\title{
"Tourette's Is a Lonely Place": an Interpretative Phenomenological Analysis of the Personal Experience and Identity of Adults with Tourette's Syndrome
}

\author{
Melina Aikaterini Malli ${ }^{1}$ (D) $\cdot$ Rachel Forrester-Jones ${ }^{2}$. \\ Paraskevi Triantafyllopoulou ${ }^{1}$
}

Published online: 14 May 2019

(C) The Author(s) 2019

\begin{abstract}
Research suggests that adults with Tourette's syndrome (TS) may face unique challenges in their everyday life due to their condition. To date, however, only a limited number of studies exist in relation to their life experiences and conception of identity. This study, which we believe to be the first of its kind, aimed to expand the literature by 'giving voice' to this population, and to evaluate the social and personal cost of living with TS during adulthood. Semi-structured interviews were conducted with sixteen adults with Tourette's that focused on how they negotiated their identity and experiences. Transcripts of recorded interviews were subjected to interpretative phenomenological analysis. Three superordinate themes emerged: a) "Incorporating Tourette's syndrome into self" revealed two divergent ways in which TS was merged into the participants' selfidentity, either by reconciling with it or fighting against it; b) "Interpersonal interaction" covered issues relating to negative and supportive ties as a result of their condition; and finally, c) "The solitude of Tourette's syndrome" described the loneliness that stems from the condition. The current findings suggest that the experience of the participants is a multidimensional one related to self-identity threat, social withdraw, and self-stigma. Implications for clinical practice on the topic are discussed.
\end{abstract}

Keywords Tourette's syndrome - Interpretative phenomenological analysis · Identity · Stigma

Melina Aikaterini Malli

M.A.Malli@kent.ac.uk

Rachel Forrester-Jones

RFJ@bath.ac.uk

Paraskevi Triantafyllopoulou

P.Triantafyllopoulou@kent.ac.uk

Extended author information available on the last page of the article 


\section{Introduction}

Tourette's syndrome (TS) is a childhood onset neurodevelopmental condition characterised by multiple motor tics and at least one phonic tic that have persisted for more than one year since their onset (American Psychiatric Association 2013). Tics are defined as paroxysmal, rapid, non-rhythmic movements (motor tics) or vocalisation (phonic tics) (Cohen et al. 2013). Motor tics range from single, short, sudden movements, such as eye blinking or nose twitching to complex behavioural sequences such as squatting, jumping, or even obscene gestures (copropraxia). Vocal tics can range from inarticulate single vocalisations to echolalia (one repeats the words of others), paralilia (one repeats his/her own words) or even the controversial feature of coprolalia (the utterance of obscenities) (Leckman et al. 2013). Tics exhibit fluctuations in frequency, type, severity and complexity within and between individuals. Knight et al. (2012) report in their meta-analysis that TS can be encountered in $0.77 \%$ of the school-aged population and in $0.08 \%$ of adults.

TS is commonly associated with other co-morbid conditions - such as ObsessiveCompulsive Disorder (OCD), Attention-Deficit and Hyperactivity Disorder (ADHD), anxiety and affective disorder - in approximately $90 \%$ of people with TS (Cavanna and Rickards 2013). Freeman et al. (2000) also document that these co-occurring conditions might affect and diminish the quality of life of individuals with TS more than the actual tics.

Studies suggest that tics follow a remitting pattern with increasing age. They usually reach their peak during adolescence and diminish in frequency and intensity by early adulthood (Bloch and Leckman 2009). Therefore, the qualitative literature hitherto has placed the emphasis on the impact TS might have on children and adolescents, and examined their lived experiences. The wider literature suggests that the complexity of TS extends beyond the physical symptoms. Social adjustment difficulties (Cutler et al. 2009), social- and self-stigmatisation (Malli et al. 2016; Smith et al. 2015), isolation, bullying, peer rejection (Malli and Forrester-Jones 2017; Wadman et al. 2013) and poor sense of self-concept due to personalisation of the illness (Hanks et al. 2016) are some of the hurdles experienced by children and adolescents affected by TS.

A sizable subset of individuals with TS however, whose symptomology persist into adulthood, exhibit the most florid clinical presentations with symptoms becoming more severe and resistant to treatment (Swain et al. 2007). They may, therefore, face unique challenges across several domains through their adulthood. Yet, individuals with persistent symptomatology during adulthood are the least studied population hitherto (Conelea et al. 2013). The few quantitative studies pertaining to adults with TS suggest that they may face greater unemployment rates, lower income, and be associated with lower socio-economic status (Aldred and Cavanna 2015; Miller et al. 2014). Additionally, they may face higher difficulties in social relationships (Conelea et al. 2013), increased familial/marital stress (Hubka et al. 1988), greater propensity for mood and anxiety disturbances (Evans et al. 2016), and ultimately a diminished quality of life (QoL) (Conelea et al. 2013). There is, however, a scarcity of research exploring the holistic impact of living with TS as an adult, the consequences of the challenges associated with the condition, and how adults with TS view themselves and construct their social and self-identity (Malli et al. 2016). By social identity we are referring to the sense of self people derive from their membership in a specific group and the emotional 
value they attribute to this membership (Tajfel 1972). Tourette's identity can be conceptualised as a social identity, one of many that can be integrated into an individual's self-concept. Research on other populations has suggested that belonging to a group can improve one's physical and psychological well-being (Jetten et al. 2012) but can also have negative consequences if the group membership carries stigma. By sense of self, self-identity or self-concept, we are referring to how adults with TS view and feel about themselves as unique individuals but also in relation to other people in their social group (ingroup members) (Tajfel 1974).

O'Connor et al. (2009) research suggests that adults with the condition appear to be concerned about the impact their tics have on others while striving to fit into the stringent boundaries of normalcy and to fulfil society's expectations. O'Connor's study however, examines representations of illness on groups of individuals with various conditions rather than focussing on TS and therefore fails to delve into the specific experiences of adults with TS. Buckser's (2008) ethnographic study indicated that the suppression and concealment of tics by people with TS appeared to be a mechanism to resist the undesirable disability label - adults with TS wishing to dissociate themselves from 'disabled' groups. Yet, self-concept and social identity were not discussed in Buckser's study and there is a gap in the literature that provides a broader understanding of the individuals' lived experience. We would argue that an exploration of how selfconcept and social identity impacts on the well-being of adults with TS is needed to help inform the provision of appropriate social support to this population.

\section{Objectives of the Study}

Despite considerable discussion concerning the implications of having TS as a child or adolescent, only a few adults have documented their personal accounts (Cohen and Wysocky 2008; Hollenbeck 1999; Mansley 2003). The experiential dimensions of TS, including how individuals conceptualise and express their identity as a person with Tourette's, have never been explored (see Malli et al. 2016) nor is there research pertaining to how adults with TS come to understand and live with their condition, and how having a diagnosis of Tourette's as an adult and being categorized into a group with other people with Tourette's affects their well-being.

Qualitative research was deemed appropriate in order to explore the experiences of individuals with TS, since it would enable an understanding of the complexities and nuances of identity. Since the manifestation of tics varies widely between individuals but also within the same person over time, idiographic and personal accounts would allow exploration of these experiences whilst at the same time capturing the essence of one individual's personal perspective of TS.

Based on the gaps in the literature, the aim of the study was threefold: (a) to examine the lived experiences of adults with TS and explore how the condition is managed in the context of their lives; (b) to investigate the personal meaning adults bestow to their condition and how they conceptualise and express their social and self-identity due to TS; and (c) to understand the impact TS has had on their social and personal relationships. By giving primacy to individuals' experiences and empowering the hitherto disregarded voice of adults with TS, we aimed to understand what it means to have TS during adulthood. 
Our study aimed to contribute to the very small body of qualitative research that gives primacy to the experience of adults with TS. We focused on identifying previously unappreciated challenges faced by adults with TS through their own accounts. In addition, the social and personal cost of living with TS in adulthood could be highlighted by exploring how adults with TS construct their identity. Finally, a fresh understanding of how adults with TS make sense of their social identity can inform clinical work with this population, which is still relatively in its infancy (Malli et al. 2016). Through this study, we therefore sought to ignite an interest in the social aspect of the condition.

\section{Approach}

Interpretative Phenomenological Analysis (IPA) was used to explore the participants' lived experiences and to gain insight into their interpretation of their personal and social world. This qualitative approach, which has theoretical foundations deeply rooted in hermeneutics and phenomenology, aims to enter the social and psychological world of the participants in order to develop an understanding of how they make sense and give meaning to their lived experiences. It focuses on an individual's personal perception of an event or state of self and not on an objective account. IPA is especially suitable for exploring topics related to self and identity, and also lends itself well to examining the psychological experience of living with a chronic condition. The nuanced and finegrained understanding that this idiographic approach uses can also provide enriched insight to under-researched topics (Smith 1996; Smith et al. 2009).

IPA aims to understand how individuals make sense of their experiences. However, it also acknowledges that the research process is a dynamic one in the sense that the researcher's interpretation and conceptions are an inevitable part of the analysis (Smith 1996). In this interpretative two-stage process, which is termed as "double hermeneutic", the participant seeks to make sense of their world, and the researcher seeks to make sense of the participant's sense-making (Smith \& Osborn 2007).

\section{Participants}

Our inclusion criteria stipulated that participants should be adults who have been medically diagnosed as having Tourette's syndrome. There was no age limit. Participants were required to be fluent in English as the interviews were conducted in English. Participants with additional co-morbidities were included in the sample except for individuals with learning disabilities, as this study was a part of a larger one investigating the social care needs of adults with TS. Individuals with learning disabilities were excluded to avoid bias in the results of the study, since they may have social care needs that might not necessarily be related to having TS.

Smith et al. (2009) recommends small sample sizes (six to eight participants) in order to allow a detailed analysis of individual accounts but also to detect patterns of similarities and differences between the participants. However, it is also emphasised that there is no 'right sample size' and researchers should feel that they have accomplished coherence and integration in their analysis, while simultaneously preserving 
nuances and presenting a suitably persuasive story (Elliott et al. 1999). To this end, the sample of this study encompassed sixteen adults with TS. Many in-depth IPA studies have employed a similar number of participants and succeeded in retaining an idiographic focus on the individual voice (Colton and Pistrang 2004; McCarthy et al. 2017). The sample included four females and twelve males. Thirteen of the participants reported being white British, two identified as other white ethnic group and one as Pakistani. Their ages ranged from 24 to 43 (mean=32.6). The mean age of symptomology onset was 8.9 years. Two of the participants reported to have experienced the onset of TS symptomology after the age of 18, three reported that their symptomology had exacerbated during adulthood, while eleven reported that their tics had stayed the same since childhood. Three participants characterised their symptoms as mild, eleven as moderate and two as severe and debilitating. Five participants had children (ranging from one to two), one of which was diagnosed or displayed the symptomology of TS. At the time of interviewing, five of the participants were receiving disability benefits due to reduced working capability mainly because of Tourette's syndrome. Table 1 outlines the demographic information of all included participants. The sample was not assumed to be representative of the broader population of adults with TS.

\section{Procedure}

\section{Ethics}

The study was reviewed and granted an ethically favourable opinion by the National Health Service Health Research Authority Social Care Ethics Committee (November 2017, 17/IEC08/0041). Informed consent in writing was obtained from all individual participants included in the study.

\section{Data Collection}

The present study was conducted as part of a larger research project investigating the social care needs of adults with Tourette's syndrome in England. Adults with TS were invited to take part in the study via websites and social media platforms, including Tourettes Action, Tourette Focus-UK/Europe and ADHD Wise UK. After taking part in an online survey, all eligible participants were asked to contact the researchers if they wanted to take part in a one-to-one interview, which resulted in a selfselected sample. All potential participants were sent an information sheet giving the details of the study and were asked to choose the most convenient medium to conduct the interview: video call, phone or in person. Fourteen participants chose to undertake the interview over the telephone, one preferred a face-toface interview, and another chose to submit written answers to the research questions via email as the participant assessed that the severity of his/her vocal tics prevented them from engaging in an interview. A mutually convenient time to conduct the interviews was organised with the first author. No participant withdrew from the study and no stress was conveyed or reported to the researchers during or after the interview. 
Table 1 Demographic Information of participants

\begin{tabular}{|c|c|c|c|c|}
\hline & Gender & Marital status & Employment status & Co-morbid disorders \\
\hline Participant 1 & Male & Single & Unemployed & ADHD \\
\hline Participant 2 & Male & Single & Paid employment & $\begin{array}{l}\text { OCD, Mood disorder, Illegal } \\
\text { prescription medication }\end{array}$ \\
\hline Participant 3 & Male & Single & $\begin{array}{l}\text { Voluntary work. } \\
\text { Receiving social } \\
\text { benefits. }\end{array}$ & $\begin{array}{l}\text { Autism, Mood disorders, anxiety } \\
\text { disorder, ADHD, OCD }\end{array}$ \\
\hline Participant 4 & Female & Single & $\begin{array}{l}\text { Paid employment. } \\
\text { Receiving social } \\
\text { benefits. }\end{array}$ & - \\
\hline Participant 5 & Male & Single & Paid employment. & OCD \\
\hline Participant 6 & Male & Married & Paid employment. & - \\
\hline Participant 7 & Male & Married & Receiving benefits. & $\begin{array}{l}\text { ADHD, Social Phobia, } \\
\text { Disruptive Behaviour, } \\
\text { Mood Disorder }\end{array}$ \\
\hline Participant 8 & Male & Cohabiting & Paid employment. & - \\
\hline Participant 9 & Male & Married & Paid employment & OCD \\
\hline Participant 10 & Female & Single & Student. Receiving social benefits. & ADHD, OCD, Social Phobia \\
\hline Participant 11 & Female & Cohabiting & Paid employment. & - \\
\hline Participant 12 & Male & Single & Paid employment. & - \\
\hline Participant 13 & Male & Married & Paid employment. & $\begin{array}{l}\text { ADHD, OCD, Social Phobia, } \\
\text { Anxiety Disorder, Mood } \\
\text { Disorder }\end{array}$ \\
\hline Participant 14 & Female & Single & Receiving benefits. & OCD \\
\hline Participant 15 & Male & Single & Paid employment. & ADHD, OCD, Anxiety Disorder \\
\hline Participant 16 & Male & Single & Paid employment & Anxiety disorder, Epilepsy \\
\hline
\end{tabular}

The participants were encouraged to seek clarification, raise any potential concerns and ask questions about the study and the research process. Data were collected over a period of seven months by the first author. Interviews lasted between 20 to $70 \mathrm{~min}$, they were audio recorded and transcribed with any identifiable information removed.

Each participant received a $£ 15$ voucher for volunteering to participate in the study, presented to them after they had completed the interview. The voucher was intended to compensate the participants for their time and effort, and to reduce non-response bias in order to achieve a sample that is more representative of the population being studied (Grady 2005).

\section{Interview Protocol}

The interview schedule was constructed using a range of previously published interview schedules relating to chronic conditions and disabilities (though no specific interviews in relation to TS were found) and their impact on social identity (Monteleone and Forrester- Jones 2017; Shinebourne and Smith 2009; Smith and Osborn 2007). Additionally, specific questions that had previously not been asked were included and the interview schedule was guided by the advisory group who 
helped to identify lines of inquiry not previously considered. Such techniques in constructing interviews have been advocated by Flowers et al. (2000) (see Table 2 for interview schedule).

In accordance with IPA, the interview style was non-directive and aimed to facilitate the participants' ability to tell their own story. The interview included topics about the emotional, social and practical implications of having TS in adulthood but also the perceived impact of TS on self and future self. Following Smith et al. (2009), prompts were used to keep the participants engaged in the conversation and facilitate further understanding. Prompts included questions such as "Can you tell me a little more about that?" and "How did that make you feel?". The interview schedule was piloted with two adults with TS that were part of the advisory group for this study to ensure the validity.

At the start of each interview, it was pointed out to all participants that they were not obliged to answer all the questions, that they were free to stop the interview at any time, and could request their data to be excluded from the analysis up until the point at which the study was completed. As with all qualitative research, there was variability in the depth and richness of the data collected within the interviews.

\section{Analysis}

Smith et al. (2009) guidelines for the process of analysis were followed. This entailed becoming familiar with the interview transcripts by repeatedly reading each one while simultaneously listening to participants' voices and making notes on tone of voice and pauses. Three types of comments were noted in the margins of the transcripts:

Table 2 Interview schedule and source of interview questions

\begin{tabular}{|c|c|}
\hline Interview Schedule & Source of Interview Questions \\
\hline $\begin{array}{l}\text { Can you tell me about yourself? How } \\
\text { would you describe yourself as a person? }\end{array}$ & $\begin{array}{l}\text { Smith and Osborne 2007, Shinebourne } \\
\text { and Smith } 2009\end{array}$ \\
\hline How did your tics start? & $\begin{array}{l}\text { Smith and Osborne } 2007 \text { (How did your } \\
\text { pain start?) }\end{array}$ \\
\hline Can you tell me what TS means to you? & $\begin{array}{l}\text { Smith and Osborne } 2007 \text { (Can you tell me } \\
\text { what pain means to you?) Monteleone } \\
\text { and Forrester-Jones } 2017 \text { (Can you tell } \\
\text { me what Intellectual Disability means } \\
\text { to you?), }\end{array}$ \\
\hline $\begin{array}{l}\text { How (if at all) has Tourette's impacted } \\
\text { different aspects of your life? Employment? } \\
\text { Social relationships? Romantic } \\
\text { relationships? Sense of self? }\end{array}$ & $\begin{array}{l}\text { Shinebourne and Smith } 2009 \text { (How has } \\
\text { alcohol affected different aspects of your } \\
\text { life? Employment? Social relationships? } \\
\text { Romantic relationships? Sense of self?) }\end{array}$ \\
\hline How do you think other people see you? & Shinebourne and Smith 2009 \\
\hline $\begin{array}{l}\text { If you could press a magic button and stopped } \\
\text { having Tourette's, would you press that } \\
\text { button? }\end{array}$ & Advisory Group \\
\hline $\begin{array}{l}\text { How do you see yourself in the future and } \\
\text { what role does Tourette's play in that? }\end{array}$ & Shinebourne and Smith 2009 \\
\hline
\end{tabular}


descriptive comments (focused on the content of the narratives), linguistic comments (focused on the participant's use of language), and conceptual comments, which entailed understanding the narratives at a more abstract level. Furthermore, extensive interpretative notes regarding how the experiences had been described by the participants and why they had been described in that way were made. These initial codes, as well as a reflective diary kept by the researcher, enabled themes to emerge. Subordinate themes were developed by scrutinising and clustering the emergent themes. In turn, the subordinate themes were clustered into superordinate themes through a process of abstraction and subsumption. Abstraction involved putting "like with like" (Smith 2009 , p. 96) and developing a name for the cluster. In the subsumption process, an emergent theme acquired a superordinate status and there appeared to be a series of other themes that fitted accordingly as subordinate themes. The superordinate themes were then compared against the original transcript to assess if it validated the participant's account as a whole. This procedure was repeated successively for each interview transcript to ensure each interview was analysed on its own merit, and to allow personal experience and insight to emerge (Smith et al. 2009). During the cross-case analysis stage, the superordinate and subordinate themes from each of the transcripts were examined to detect patterns across cases, with attention paid to both convergent and divergent themes. NVivo 11, a qualitative analysis software, was used to manage the data.

\section{Validity}

Although the authors acknowledge that alternative interpretations of the data are possible, special care was taken to ensure the validity of this research. Smith (1996) suggests that we should not evaluate qualitative research with quantitative criteria; rather, different criteria are advocated which entail internal coherence and presentation of evidence. Internal coherence refers to whether the research is internally consistent and whether it deals with contradictions and ambiguities, and if it offers alternative readings. Presentation of evidence dictates that ample raw data should be available so that the reader can interrogate interpretations being made. In this article, the transcript extracts allow the reader to evaluate the interpretation made by the researcher.

Furthermore, Lincoln and Guba (1985) suggest reflexivity as an indicator of validity. The researcher acknowledged that complete objectivity was not possible but aimed not to allow personal values to skew the research. This was achieved through a reflective journal that was kept during the interviews, transcriptions and data analysis (Rossman and Rallis 2003).

Guba and Lincoln (1989) propose member checks as a method to enhance the credibility of a qualitative study. The checks can take place "on the spot" during the course and at the end of the interviews. This method was used to assess the accuracy of the data.

Finally, half of the transcripts were independently coded by the second author to ensure validity. Any disagreements about interpretations were discussed until an agreement was reached. In line with the IPA guidelines, inter-rater reliability coding did not aim to produce a categorical analysis but rather to verify that the analysis had been systematically conducted and was supported by the data. 


\section{Results}

The delineated themes are presented and accompanied by substantive anonymous quotes from the participants, which have been unaltered by the authors to maintain the participants' authenticity. The quotes selected were evaluated as the most representative, and the ones that articulated and exemplified the core essence of the themes. They were also picked based on their efficiency to demonstrate both the variation and depth of a theme. Three dots indicate a pause in the participants'speech, empty square brackets indicate omitted or explanatory material. Identifying information is replaced within square brackets.

Three distinct yet interconnected superordinate themes emerged from the data analysis: "Incorporating Tourette's syndrome into self", "Interpersonal interaction" and "The solitude of Tourette's syndrome". Each of the superordinate themes comprised of at least two subordinate themes. All of the subordinate themes were evidenced with data from at least half of the participants in line with the recommendations of Smith et al. (2009). The recurrence of subordinate themes in at least half of cases was a way to enhance the validity of the findings.

\section{Incorporating Tourette's Syndrome into Self}

Two distinct and divergent groups were generated in relation to incorporating TS into self: individuals that had accepted their condition as part of their own identity and life, and were willing to work with it as opposed to against it; and those that felt inferior due to TS and were grieving the loss of normalcy. The former group described themselves in positive terms, contrasting acutely to the participants who felt that Tourette's had debilitating consequences for their sense of self. The latter group felt restricted by TS and were incapacitated by the stigma that the condition attracted, and were dissatisfied with their existence. Unlike those people who were living with Tourette's, this group was trying to live through Tourette's syndrome.

\section{Living with Tourette's Syndrome}

TS had become an indispensable part of the life and reality of this group. As Sacks (1995) had characteristically mentioned for the case study of the surgeon with the condition, which he so vividly described, TS no longer stood "next to, but conflated with the I" (p.73). They had grown to accept and reconcile with their differences. Thus, although the underlying awareness of TS remained, they were determined to live meaningful lives.

"Because Tourette's is me and I've obviously come to terms with it and, you know, I embrace it...and, you know, I've learned to accept that that's just me.... I don't know if embracing is the correct word...but, you know, I've learned to accept that as part of my...of who I am." (Participant 9)

Tourette's was viewed as another aspect of their identity that rendered them different to their peers but was not an all-encompassing identity. Thus, the 
stigma had not managed to become what Goffman (1963) termed as their 'master status' and did not deduce who they were to a label surrounding their Tourette's. Simultaneously, it was acknowledged that homogeneity was untenable and that every individual possesses attributes that renders them unique. In that sense, TS was no different to their peers' unique characteristics. Participant 8 characteristically mentioned:

"Everyone has an issue mentally or physically...so...it's just mine you can see them."

Participant 13 expressed a greater level of acceptance and presented TS as an attribute that did not fully define his identity or his life. He still viewed himself as a unique multidimensional person and was able to distinguish between his impairment and selfidentity:
"It's a small part of the whole. This is a small part of what you are. As much as, say, someone who picks their nose and then hides it behind the sofa or something. It is as much as that as one hobby. Humans can't be defined by just one aspect... a part, there are a lot of things about you. So, it's just a part of, not the defining whole"

The condition was never who one was but only something that a person had. In other words, the participants did not perceive Tourette's to be self-defining and did not internalise it as a salient part of their social identity. This was achieved by making social downward comparison, through focusing on other people with TS who were worse off than themselves (Festinger 1954). The comparison was usually done on dimensions of tic severity and achieving life goals. They were therefore inclined to see themselves in a positive light through a self-enhancing social comparison. This could be interpreted as an identity management strategy adopted in response to the stigma attached to TS.

\section{"I think perhaps their level of Tourette's was much worse than me" (Participant 11) \\ "I consider myself quite lucky in a way because I'm able to carry on with most patterns with almost a life experience of it. I guess I feel comfortable with it, but maybe other people [with TS] are still trying to come to terms with it, or have it much worse and stuff" (Participant 12)}

Some participants also believed that the condition had afforded them an opportunity for self-development and felt that it let them evoke a search for a greater meaning in their lives. By facing adversity, they had managed to become emotionally stronger and TS allowed them personal integration and growth.

"It sounds really cheesy, but I think it [TS] has made me a better person and it's made me want to help other people" (Participant 3)

"It actually gave me more focus to try and help those who do have it [worse than I do] which is what I want to do. But, also, it also kind of helped me get into 
support work to deal with people with learning disabilities. So, it enhanced my desire to do things like that" (Participant 13)

\section{Living through Tourette's Syndrome}

Individuals in this group talked at length about how TS had taken things away from them, assumed centrality in their lives, and defined their sense of self. A discourse of loss, disadvantage and anger was communicated by these adults who viewed TS as being in charge of their being. Most importantly however, TS deprived them of their normalcy. TS was an identity that they had not embraced and there was a clear dissonance between their preferred social identity and the persona that TS imposed on them. These interviewees were not living with the condition but 'trying to live through it.'

The participants descriptions of themselves and their Tourette's syndrome was characterised by negative valence notions of difference that held them back from achieving their life goals:

"I feel that my Tourette's prohibits me from doing so much. And I have so much to offer. But unfortunately, I can't do what I need to do because of my Tourette's." (Participant 7)

Participant 15 vividly described how TS had deprived him of normalcy while also revealing an underlying anxiety about how the condition left him vulnerable to external hostile judgement. There is a sharp distinction between other peoples' reality and his own in which he struggled with his condition in a personal and interpersonal level.

"It's a disorder that just makes me, separates me from the rest and in my eyes, it's quite a big distance, because I don't feel as though I can live a normal life most of the time with it. And it's not even just things that people can see on me when they first meet me. They are the things that happen behind closed doors that no one sees and only I see, or sometimes don't see."

The rejection of the TS-categorisation may also be due to the fact that participants belong to other significant groups to which they draw positive self-esteem from. The hypervisibility of Tourette's and the physical consequences of it made them want to dissociate themselves from it.

It's really not something I would like to have. I have a diagnosis for autism spectrum disorder and I'm quite...not proud of that, but I embraced it. But the tics, I do not like them. I feel very, very uncomfortable a lot of the time, I feel like I can't relax and the sensation just before, it makes me very, very uncomfortable. It drives me crazy. And I find it very embarrassing and I don't want the attention... (Participant 3)

In response to the TS stigma, many participants tried to conceal their spoiled identity (Goffman 1963) by trying to control their tics. This helped them maintain a socially 
desirable identity. In other words, due to their ability to control their symptomology, the boundaries of the TS group were viewed as permeable:

"I'm quite good at controlling it, I'm quite good at hiding it and getting out of situations...At the moment not a lot of people would necessary have seen it. Only people close to me or people that see me on a regular basis really" (Participant 4)

Medication (usually antipsychotic drugs also used to treat schizophrenia) was reported by participants as an effective means of concealing their tics. Nevertheless, most participants reported that the side effects of medication were distressing and deprived them of their personality:

"I lot of the medication that I've tried have just made me feel like rubbish. Like haloperidol... and that will make you feel like you're a zombie. I am naturally like a very lively, active person and they just make you feel like you've got no energy and you feel like...it's rubbish." (Participant 11)

In response to their spoiled identity, the participants also tried to overcompensate for their tics by bolstering other socially desirable aspects of themselves. This is a common strategy among members of stigmatised groups in order to maintain a positive selfimage and disprove stereotypes that are commonly linked to their social identity (Miller et al. 1995; Forrester-Jones and Barnes 2008). As participant 8 illustrates, having TS may mean working to standards that exceed conventional ones in order to win other people's respect, avoid prejudice and prove that they are as good as their non-TS peers.

“....if everyone's doing 100\% I will do 130\%. I am the one that does the most work, so... and that's always the case, cause if they're gonna get rid of anyone, they'll get rid of the person who twitches, cause he's, annoying."

Overexertion however, although effective temporarily, may "drain the individual's resources" (Miller and Major 2000) and ultimately have long-term negative effects on the individual's health.

The sense of loss was even more pertinent to the minority of participants who developed their symptoms at an older age (over 18 years old). These adults had to go through the process of biographical disruption and reconstruction (Bury 1982) as opposed to individuals who developed TS from an early age and therefore were socialised to their role from quite early on. They discussed a dichotomy between the 'old self' before the TS and the one after. The latter was infused with altered social relationships and altered visions for the future. They described their lives as going through a qualitative shift and their past happiness, normalcy, self-sufficiency was contradicted to their current situation:

"It has completely messed up my life to put it politely. If less severe, that would be difficult at times but at least I could still do things. I feel fortunate that when I was young I had no tics and for many years, they weren't severe enough to cause too many problems, but at this severity I struggle with it. So, I did have a busy life 
back then, working hard but also having lots of fun, travelling all over the world." (Participant 14)

\section{Interpersonal Interaction}

In their accounts, the participants made extensive reference to social interactions they had throughout their lives and the subsequent impact these had on their self-esteem. Both negative and positive relationships were described.

\section{Negative Social Interactions}

Most of the interviewees described how their symptomology was discredited, commenting on the negative feedback from their parents, teachers and siblings as a regular occurrence since childhood. Their tics were dismissed as simply behavioural problems in the past, which led to negative judgement by others.

"They said [doctors] that I was just playing up and that I was to be told off every time I twitch" (Participant 8)

Nearly all of the participants in the study reported some form of rejection or victimisation as a response to their condition and described the subsequent effect that had on their self-esteem. For some, victimisation and marginalisation was not only encountered within the realm of the public arena but also within their homes growing up. Family conflict and poor communication was narrated by many participants who felt criticised for their condition. They reported on how their families attributed blame to them, as well as a lack of parental understanding and acceptance. For some, this resulted in parents placing unreasonable demands on individuals with TS:

"My parents ...they were horrible to me. They thought by telling me off or slapping me or whatever, punishment, you know, name calling...they thought that I would stop doing it. They thought it was something, you know, that could be stopped. So, ended up like watching television together and back in the days we had one television. So, I would have to sit behind the family, so they wouldn't get distracted. And they would humiliate me in public, you know, they would say stop being a monkey and then my sisters obviously because they were... they saw my parents having a go at me and bullying me for that they would do exactly the same" (Participant 9)

As is clear from most participants' accounts, the parents, due to their lack of awareness and understanding about the condition, assumed the role of what Schneider (2009) term as 'stigma coaches'. Thus, they taught their children that their tics represented an undesired differentness or a moral weight they had to carry. They solely focused on the restrictions faced by people with tics and coached them to conceal their condition from others. Similar to parents of people with intellectual disabilities, close family members of our participants were the key people in shaping their 
identities and providing them with the social meaning that the condition bears (Todd and Shearn 1997).

Discrimination did not always manifest overtly but the participants also described subtle behaviours of the stigmatisers. They reported that people may respond to them with either superiority or excessive politeness.

"They [employers] kept looking at me and smiling, like I was a helpless puppy... I think they didn't mean any harm, but they were very patronising, surprised that I even had the qualifications to apply for the job" (Participant 1)

This phenomenon, which Link and Phelan (2014) term as "interaction discrimination", is parallel to racial microaggression. It has been attributed to less public tolerance of overt displays of prejudice. Thus, it is a form of subtle, unconscious and mostly unintentional degradation of a group in everyday interaction. Because they often occur outside the level of conscious awareness, well-intentioned individuals can engage in these biased acts without guilt or knowledge of their discriminatory actions (Sue 2003). Furthermore, the subtle tone of these interactions means that they are difficult to document and often dismissed and glossed over as being innocent and innocuous. However, they can have a detrimental impact on an individual's self-esteem.

Victimisation had led to the internalisation of the "socially odd" image that had been ascribed to them by the stigmatisers. Low self-confidence, poor self-esteem stemmed from these interactions and restricted them in their everyday life:

"I feel like really sort of embarrassed, almost ashamed. They made me feel it was my fault, that I was doing something wrong and it also kind of made me feel segregated, always...like I fit in a different box." (Participant 3)

Interviewees also indicated through their narratives how the aftermath of being victimised still manifested in their social relations. Participant 15 described a lingering internal fear of becoming a victim again.

"I don't want to bear the brunt of it, like I was when I was in school. It was a bad experience and I'm dead scared of that happening again."

The negative social interactions that the participants may have experienced could have a detrimental impact on the self-consciousness. Indeed, as Cooley (1922) mentioned in his looking-glass self-theory, "we live in the minds of others without knowing it" (p. 208). Therefore, receiving negative feedback could give rise to intense emotions of shame.

\section{Supportive Relationships}

Some participants described a supportive social network that provided a buffer against the outside world (Forrester-Jones and Grant 1997). Indeed, a non-judgemental and empathic group of friends was depicted that shielded them from negative experiences, discrimination and stigmatisation. Participants described finding acceptance in spite of a limiting condition, and they subsequently deepened relationships with those who 
provided such understanding. This social support advocated on their behalf when necessary.

"If I'm with my other friends with TS, I feel quite OK to tackle social situations and be myself." (Participant 4)

Other participants also described the moral, financial, practical and ethical support their significant others provided.

"One thing that my partner does that really helps actually is, one of my tics is whistling and for example if I start to whistle in public and I feel embarrassed he will whistle along with me. And then I don't do it alone." (Participant 2)

"If I'd had been single I think it would have been necessary [to apply for benefits] because, you know, I would have needed to have some money at the time" (Participant 3)

A participant identified their partner as their 'number One', indicating their supportive emotional role.

In the presence of those intimates, the participants could drop their mask and step out of character whilst their partners had access to what Goffman refers to as 'backstage' (1959), in which suppressed behaviours and the otherwise condoned upon tics could be expressed. These intimates represented the wise, the normal who could relate to the stigmatised individuals.

\section{The Solitude of Tourette's Syndrome}

This theme encapsulates the deeply isolating consequences of having TS as an adult. As participant 5 quite aptly mentioned "Tourette's is a lonely place". The participants described a feeling of loneliness that stemmed from being segregated from the normal, and a lack of understanding in society, but also emerged from self-isolation and social withdrawal to cope with enacted stigma. Lack of readily available support and resources for adults with Tourette's syndrome also contributed to their profound sense of social isolation.

\section{Involuntary Isolation}

Participants' childhood and adolescent memories of being singled out, victimised or mistreated because of their TS are particularly poignant examples of how involuntary isolation occurred.

"I was kept in the...I was hidden in the cupboards and the rooms. I was never taken out into public. I was even kept away from my own family except from my grandparents" (Participant 7)

Isolation also manifested by being ignored or overlooked in social settings. 
"People either are rude to me or they ignore me. Often, often they speak to the social worker, and ignore me even if explained to them that I have TS, can understand and communicate via a speech device" (Participant 14)

The excerpt above indicates the social invisibility that this group might endure and a resulted feeling of isolation.

Segregation for people with TS was also implemented through excluding them from the workforce and therefore depriving them of the opportunity for full participation in the labour market. Participants described how, from their viewpoint, employees failed or refused to make reasonable adjustments which in turn inhibited them from becoming employed or sustaining a job.

\begin{abstract}
"Imagine in pubs and working in a bar and stressful nights and stuff like that and you start ticking and people start asking questions, poking fun at you drunk, so, you know...And you try and speak to your employer about it... 'I need to stop right now, I need to pull myself away or go home or something because it's gonna make me worse'. It's just a big blow down, it really is. And they don't understand that, and they don't care either and they don't believe they have to care" (Participant 14)
\end{abstract}

\title{
Social Identity Threat and Social Withdraw
}

Most interviewees expressed a concern about being devalued and discriminated against because of their tics, and being judged through the lens of a negative group stereotype. This fear, which may have been triggered by past incidents of stigma or even by anticipation or suspicion of prejudice, may lead to social identity threat (Steele et al. 2002). Indeed, social identity threat can be perceived as a "threat in the air" that stems from being aware about the negative collective representations of TS. Participant 15 explains:

"I try not to tic in front of them, I try to hide it the best way that I can. I just feel that way, that people judge and I can't escape that for some reason"

The above excerpt clearly illustrates the individual's concern about being judged solely based on their devalued identity and not on their individual merits or abilities.

In response to social identity threat, individuals in the study appeared to avoid situations that would expose them to the prejudice of others. Indeed, the majority of the participants believed that they would be devalued and rejected by others, causing them to withdraw socially:

...the tics really made me less likely to reach out socially. Well, to socialise at all or to seek out friends, cause I was worried about being the joke (Participant 3)

Social avoidance may be viewed as a protective strategy to tackle social exclusion and feelings of rejection. Some of the participants described the discomfort they felt when in the presence of others. In response to social identity threat, the participants preferred 
to retreat into the company of a few selected friends who understood their condition. There was a clear avoidance of disclosure, anxiety about exposure and vigilance to others behaviour. Participants appeared to be troubled about others' reactions to their condition and this led them to actively avoid social interaction and self-isolate. As participant 10 explains, it appeared easier for her to withdraw from the public view than to rely on the understanding of others:

"Well, as a direct result of Tourette's I have social phobia, social anxiety. So I don't really integrate with people very well. It's embarrassing...that's why I tend to stay away from people, because I'm more consciously aware all the time that... not wanting to tic in front of people because people will not really know what's going on and it's just...it's humiliating. It's just...I'll go for avoidance rather than, you know, tackle it head on."

"I'd rather stay at home and not put myself in situations with other people. I definitely don't go to many, like, social events or I hate meetings" (Participant 4)

This is in accordance with Goffman (1963), who argued that stigmatised individuals begin to self-isolate because society fails to grant them the respect they anticipate.

"Maybe I would hold off on doing certain things or being in certain places because of my Tourette's or fit in certain places to avoid attention or distract other people. Like for instance cinema I would get the back seat." (Participant 9)

In this study, participants' responses to discrimination served to further socially isolate them, and in turn, perpetuate the cycle of stigma.

\section{Lack of Resources for Adults with Tourette's Syndrome}

Many participants pointed out that there was a lack of social resources for adults with Tourette's syndrome. Although they maintained that social and emotional support was available for children with TS and their parents, resources for adults were limited and could not be easily accessed. Whilst TS is not a condition restricted to childhood, the participants asserted that there was no continuity of care and that individuals with TS were at a high risk of failing to transition successfully to adult services.

"At the age of 16, I was just pushed over the edge and told to get on with life. I've not had any inquiries or contact from anyone regarding it...since the age of 16. That was it. That was the end of my care as far as anyone was concerned" (Participant 15)

In addition, participants felt that their social support needs were not adequately addressed. They did not have access to support groups that were specifically for adults with TS, and subsequently, rarely had the chance to meet and befriend other adults with the same condition who might have lived through similar experiences and might be able to provide them with information about the condition. In other words, they had no opportunity to meet what Goffman (1963) refers to as "own", stigmatised individuals 
who share a particular devaluation and are able to comfort others who have the same identity. Participant 9 clearly pointed out:

"A lot of information they [relevant organisations] were providing was mainly geared towards children with Tourette's syndrome and parents that have children that have Tourette's. And there was not much for adults, so I have to say...I had to figure out a lot of things all by myself"

Many of the participants maintained that access to an adult group would decrease their sense of isolation, would allow them to be part of a community, and give them a sense of belonging, a sense of safety and unconditional acceptance. Furthermore, it would give them a platform where they could freely discuss their condition, their feelings and strategies to cope with TS.

“..although, well it's nice to be unique, I would always like somewhat, you know, a group of people, or someone I can talk to about, you know, or associate with and I feel I'm like...I'm part of something almost." (Participant 13)

\section{Discussion}

The study investigated a group of adults' experiences of living with TS and how the condition had impacted their social identity and sense of self. Qualitative methodologies and, more specifically IPA, enable stories to be told directly from the people who have had the experiences and gain insights into some of their perceptions, something that has previously been lacking in this particular field.

In relation to exploring the lived experience of adults with TS, our study indicated that the participants encountered both supportive and unsupportive social relationships as a response to their condition. However, the participants felt that the social stigma linked to the condition isolated them. In addition, the fear of being discriminated against caused them to withdraw from social interaction. In relation to social-identity, our findings suggest that the "TS-identity" and group membership had been rejected by most participants.

\section{Self- Identity and Tourette's Syndrome}

Our study suggests that individuals with TS tend to incorporate the condition into their identity in two ways: by trying to work with it and minimise its influence in their lives, or by working against it. The former group had accepted TS and had learned to live with it; it was inconsequential to their self-identity and not central to their self-concept. This was mainly due to the fact that these participants perceived the differences between other people with TS and themselves as more salient than the differences to individuals who did not have TS and were members of other groups. Indeed, as is evident from the findings, participants with TS that had accepted their status were keen to make self-enhancing downward comparison to other individuals that had TS (Wills 1981; Monteleone and Forrester- Jones 2017). They compared themselves to individuals that had more pronounced or severe symptomology and therefore viewed 
themselves as occupying a more favourable position than less fortunate others with TS. By focusing on similarities with outgroup members and on the differences with ingroup members, their "TS identity" was not considered salient and was not integrated in their self-concept (Turner and Tajfel 1982).

The group working against the condition described feelings of difference as being central to their understanding of TS and wanted to distance themselves from the condition. With little or no positive representations of individuals with TS, it is not surprising that many participants reported emotional difficulties in their identity construction. In both groups, the participants did not view TS as a dominant identity characteristic.

\section{Social Identity and Tourette's Stigma}

As is clear from the data, the participants did not embrace TS as a positive social identity and distinguish themselves from other people with the same condition. They refused to view TS as a valid identity mainly due to the stigma associated with the condition. Rejecting or denying group membership is a common mechanism for people that belong to stigmatised groups in order to avoid prejudice (Finlay and Lyons 2005; Meisenback 2010).

\section{Stigma Derived from Group Membership}

Stigmatisation and devaluation, as a result of their condition, was prominent in the participants' narratives in relation to social interaction. In many descriptions, the home was a key site, where rejection or the fear of bullying was a regular part of life. These findings resonate with previous literature in which parents of individuals with TS tried to discipline their children, sometimes even by using violence due to their lack of understanding about the condition (Khoury 2010). It is therefore important for professionals not to solely concentrate on ameliorating the tics of the affected person but also to provide support for the whole family. Delivering psychoeducation to parents in order to manage their child's symptoms is of great importance since it can endorse positive attitudes and healthy interactions within the family (Himle et al. 2018). Frictions with parents and siblings of an individual with TS suggests that the impact of TS extend beyond the individual and may affect family functioning.

Apart from overt discrimination, a subtle demeaning message was communicated to people with TS due to less public tolerance of overt displays of prejudice. Because of its convert nature, it can be misrecognised as an innocent and trivial act. Nevertheless, it is common towards stigmatised groups and it can lead to a sense of heightened helplessness for these individuals, denying them their privacy, identity or disability experience (Sue 2010). Future research should therefore further explore how microaggression is applied to individuals with TS and how it could be ameliorated through relevant interventions.

\section{Defensive Behavioural Strategies to Cope with the Stigma of Tourette's Syndrome}

The findings of the study indicate that the participants may be vulnerable to experiencing social identity threat. Thus, due to being aware that TS is a culturally devalued 
condition, adults with TS may anticipate discrimination. This fear could arise in response to previously experienced incidents of stigma but also from the anticipation of being devalued. The participants' reaction to the threat of being rejected or devalued was to minimise the situations that could expose them to the prejudice of nonstigmatised others in public. This is considered a defensive behavioural coping strategy and it may serve as an adaptive mechanism insofar as it reduces distress and limits exposure to discrimination (Ilic et al. 2014). However, social withdraw also means one may have to rely less on their dwindling social resources and limit the diversity of their social network. Studies suggest that limiting one's social interaction leads to poor mental health and ultimately a diminished quality of life (Miller and Kaiser 2001). It also prevents adapting to stigma stressors and bolsters the feeling of inferiority. Indeed, the study indicated that most of the participants had a small, core network comprising of relatives, close friends or romantic partners that provided social support and were very involved in their everyday life. They provided a social safety net, helped them manage TS and reduce its fallout. However, their social networks were mostly lacking of peripheral (weak) ties due to stigmatisation and social withdraw. Weak ties are unstable and sparse but nevertheless enhance the quality of life of an individual, help their social integration, and mitigate loneliness (Fingerman 2009). Furthermore, they can provide a diverse viewpoint, useful information and offer greater objectivity resulting from less emotional attachment between the members.

Another defensive behavioural strategy that the participants used in order to avoid the stigma experience is overcompensating. To enhance their social desirability, they excerpted more effort than non-stigmatised individuals and believed that they had to work harder in order to receive the same evaluations as them and strive to be successful.

Many adults with TS in this study wanted to maintain a socially desirable identity by suppressing the tics and concealing their group membership, or by avoiding social interaction. They therefore believed that they could move from their stigmatised and devalued status and escape the discrimination their group may face by "passing" as normal. It is common for members of stigmatised groups to disidentify with their counterparts and align their behaviour with the non-devalued community as a means of coping with stigma (Tajfel and Turner 1979). For example, the study of Crabtree et al. (2010) illustrated how individuals with mental illness might distance themselves from the stigmatised group to avoid negative consequences for their self-esteem. However, tic suppression has been reported to be a distracting, demanding and attentionconsuming task that can create post-suppression discomfort and it is often of limited duration (Cutler et al. 2009; Matsuda et al. 2016). A substantive amount of literature also suggests that identity-concealment may lead to worse mental health outcomes, including greater levels of anxiety, depression and lower selfesteem (see Quinn et al. 2014).

As is evident, the techniques the participants used to tackle stigma were nonassertive, in the sense that that they did not directly address discrimination but avoided it, were overcompensatory in order to escape from it, or were geared to concealment of identity (Chung et al. 2009). Individuals were internally coping with prejudice and, as they identified the problem within themselves, were apologetic about it. Defensive and 
self-directed strategies to avoid or mitigate the impact of discrimination is common among stigmatised groups (Major and Schmader 2018). This is done by stigmatised individuals accepting the devaluation directed at them and finding ways to carry on without affecting the mainstream culture. There is evidence to suggest that this coping mechanism may impair an individual's self-concept because it inadvertently limits social opportunities and induces psychological distress on the stigmatised and ultimately exacerbate the harmful effect of stigma (Link et al. 1991). Nevertheless, future studies need to assess the effects of these strategies on the well-being and self-concept of adults with TS.

The defensive mechanisms that the participants adopted also reveal selfstigma. Indeed, common expressions of self-stigma include feelings of shame, which reveal that the participants endorsed cultural stereotypes in relation to TS, considered that these applied to them, and believed that they would be devalued. Therefore, more interventions need to be developed to eliminate selfstigma among adults with TS.

\section{Dissociating oneself from People with Tourette's Syndrome}

None of the participants in the group tried to counteract the discrimination of TS by increasing identification with the TS group. According to the rejection-identification model, stigmatised individuals can attenuate the negative effects of discrimination and maintain their psychological well-being and self-esteem by enhancing their group identity (Branscombe et al. 1999). The participants' decision to distance themselves from the stigmatised group may be due to the fact that they were not aware of adult specific Tourette's support groups which prevented individuals to identify with groups whose members share the same devalued status of TS. Thus, participants had no groupbased buffer against the negative effects of belonging to a stigmatised group, which a support group could potentially offer. Indeed, support groups could help increase group identification and cohesion, help build a sense of pride for individuals with TS and help them engage in collective action against discrimination. The participants of the study pointed out the need for better resources for adults with Tourette's syndrome, and suggested that adult specific Tourette's support groups could forge a unique sense of community and unconditional acceptance between members with commonalities. They also maintained that their sense of isolation and rejection could be ameliorated in a TS community for adults that would foster feelings of belonging among their members. Previous research on the benefits of support groups in coping with stigmatised conditions highlight the importance of providing a safe space where individuals could express their fears, and receive support and advice (Crabtree et al. 2010). Support groups have also been acknowledged as sites of unique reciprocity and mutuality that could facilitate expansion of social networks and understanding of self.

The fact that a collective voice has not been formed also has implications for the self-advocacy movement. Indeed, there appears to be a lack of self-advocacy groups in relation to TS, and protest against discrimination is still individual and disorganised. Research suggests that membership of these groups can provide an opportunity for empowerment, counterbalance some of the negativity derived from stigma and ultimately can promote changes in society as a whole (Anderson and Bigby 2017). 


\section{Limitations}

The findings of this study must be considered in the light of a number of methodological limitations. The majority of the interviews were conducted over the telephone or by email. Previous literature has pointed out the methodological limitations of conducting semi-structured qualitative interviews by telephone (e.g., inability to 'read' the participants' visual cues, poorer rapport with interviewees) and asserted that it might not be an appropriate medium on which to ask sensitive questions. Nonetheless, in comparison to face-to-face interviews, telephone interviews are less intrusive for participants with TS, allowing increased privacy, that could result in a more enhanced interviewer-interviewee relationship - both elements important to generating rich qualitative data. They are also more pragmatic and allow participants that live far away and those with mobility difficulties to take part in research (Holt 2010). Finally, they have been used successfully in IPA research in the past (Reilly et al. 2008). Respectively, the use of e-mail interviews has been found to be a particularly effective method of including persons with disabilities in the research process who might not otherwise be willing to participate (Murray and Sixsmith 1998).

The sample of adults with TS were a heterogeneous group. IPA aims to recruit a homogeneous sample and, although this was our initial intention, there was a variation of years of lived experiences and severity of symptomology as well as co-morbidities. Participants had been living with the condition for varying amounts of years and two individuals had developed tics after entering into adulthood. Following analysis, it became apparent that there was a qualitative difference in the psychological impact of TS based on the participants' severity of symptomology and age of onset.

Participants' diagnosis were not confirmed by accessing medical notes and information regarding their TS was obtained solely by self-report. Furthermore, two of the participants did not fit into DSM's (Diagnostic and Statistical Manual of Mental Disorders) stringent diagnostic criteria, which stipulate 18 as the upper age of onset (American Psychiatric Association 2013). Yet, in ICD-10 (World Health Organization and WHO 1992), which is most commonly used in the UK, there is no specific criteria that exclude de novo adult onset tic disorder from being characterised as TS.

Although there is a male preponderance of TS among children and adolescents, among adults, the male preponderance has been found to be less pronounced, with women representing up to $35 \%$ of the population (Freeman et al. 2000). This suggests that the female population in this study was underrepresented. Nevertheless, the experiences of women appeared to be in alignment with the male ones.

In relation to the first author's variables, her experience as a Support Group facilitator for people with TS may have affected the interview schedule and the subsequent analysis. Yet, her previous interaction with people with TS might have also helped her find nuances and enabled in-depth understanding that may have been impossible in the absence of these experiences. Nevertheless, none of the researchers experienced TS themselves and this realisation made them more diligent in the data analysis process and heightened their awareness of potential biases (Pillow 2003) with steps taken to resolve these risks. 


\section{Conclusions and Recommendations}

The themes that were generated highlight the multidimensional experience of having TS as an adult for the participants of this study. Rather than simply a neurodevelopmental impairment, TS is clearly a social condition that can also be laden with a sense of shame and loss. As this was the first study of its kind, the importance of the findings has international implications that exceed the UK borders and knowledge gained from it should help to inform healthcare professionals about the complexity of having TS as an adult and enable more effective engagement with this population. Indeed, understanding the difficulties adults with TS face in their everyday life can help clinicians as well as social care practitioners develop and support effective coping strategies that extend beyond simply minimising the tic severity. The condition, therefore, should be approached more comprehensively. Clinicians should be aware that adults with TS might invest significant energy into trying to conceal their tics. This 'camouflaging' could have detrimental effects on their psychological well-being as seen in other populations (see Cage and Troxell-Whitman 2019). Clinical approaches should aim to facilitate the development of positive Tourette's identity and focus on the positive traits associated with the condition. Due to the stigma attached to TS, individuals with the condition should be offered psychoeducation/supportive therapy to educate them about TS, as well reduce their anxiety and enhance their self-efficiency. Self-stigma can also be ameliorated through therapeutic interventions such as cognitive behavioural approaches, which can be effective in challenging irrational selfstigmatising beliefs, and replacing them with more rational and positive beliefs (Lucksted et al. 2011). However, the general public should also be approached to ameliorate social stigma. Anti-stigma interventions that aim to combat TSdiscrimination and prejudice should be used to educate and raise awareness about the condition. Social care policy has hitherto been almost silent on the topic of TS and future research should place the onus on understanding the social care needs of adults with TS. Given the complexity of experience of TS that this study has unearthed, it is perhaps time for more nuanced policies to be written. The right not to be discriminated against, as well as access to health and social care are some of the rights that might be violated in this population (see UK Human Rights Act (HRA) 1988 and The Convention on the Rights of Persons with Disabilities (CRDP), which the UK signed up to in 2009. There is currently no specific policy guidance that safeguards the human rights of this population to ensure their inclusion in wider society. Policy that aims to improve the quality of life of people with TS, which mirror white papers for people with for example, learning disabilities (see Valuing People (HM Government 2001) and Valuing People Now (HM Government 2009)), need to be developed for people with TS. Although it is acknowledged that TS is considered a distinct neurological condition, people with TS can account for up to $1 \%$ of the population (Knight et al. 2012). This makes the condition almost as prevalent as Autism (Baron-Cohen et al. 2009), signifying the importance of instigating specific policy guidelines for this population.

Acknowledgements We thank Tourettes Action for advertising this study and their support in the recruitment of participants. 
Funding This work was supported by the National Institute for Health Research (NHIR) School of Social Care Research (SSCR) under Grant C088/CM/UKJB-P118. The views expressed in this publication are those of the author(s) and not necessarily those of the NIHR SSCR, NHS, the National Institute for Health Research or the Department of Health.

\section{Compliance with Ethical Standards}

Ethical Approval All procedures performed in studies involving human participants were in accordance with the ethical standards of the institutional and/or national research committee and with the 1964 Helsinki declaration and its later amendments or comparable ethical standards.

Informed Consent Informed consent was obtained from all individual participants included in the study.

Conflict of Interest No potential conflict of interest was reported by the authors.

Open Access This article is distributed under the terms of the Creative Commons Attribution 4.0 International License (http://creativecommons.org/licenses/by/4.0/), which permits unrestricted use, distribution, and reproduction in any medium, provided you give appropriate credit to the original author(s) and the source, provide a link to the Creative Commons license, and indicate if changes were made.

\section{References}

Aldred, M., \& Cavanna, A. E. (2015). Tourette syndrome and socioeconomic status. Neurological Sciences, 36(9), 1643-1649.

American Psychiatric Association. (2013). Diagnostic and statistical manual of mental disorders (DSM-5®) American Psychiatric Pub.

Anderson, S., \& Bigby, B. (2017). Self-advocacy as a means to positive identities for people with intellectual disability: 'We just help them, be them really'. Journal of Applied Research in Intellectual Disabilities, 30, 109-120.

Baron-Cohen, S., Scott, F., Allison, C., Williams, J., Bolton, P., Matthews, F., \& Brayne, C. (2009). Prevalence of autism-spectrum conditions: UK school-based population study. British Journal of Psychiatry, 194(6), 500-509. https://doi.org/10.1192/bjp.bp.108.059345.

Bloch, M. H., \& Leckman, J. F. (2009). Clinical course of Tourette syndrome. Journal of Psychosomatic Research, 67(6), 497-501.

Branscombe, N. R., Schmitt, M. T., \& Harvey, R. D. (1999). Perceiving pervasive discrimination among African-Americans: Implications for group identification and well-being. Journal of Personality and Social Psychology, 77, 135-149.

Buckser, A. (2008). Before your very eyes: Illness, agency, and the management of Tourette syndrome. Medical Anthropology Quarterly, 22(2), 167-192.

Bury, M. (1982). Chronic illness as biographical disruption. Sociology of Health \& Illness, 4(2), 167-182.

Cage, E., \& Troxell-Whitman, Z. (2019). Understanding the reasons, contexts and costs of camouflaging for autistic adults. Journal of Autism and Developmental Disorders, 49, 1899-1911. https://doi.org/10.1007 /s10803-018-03878-x.

Cavanna, A. E., \& Rickards, H. (2013). The psychopathological spectrum of Gilles de la Tourette syndrome. Neuroscience \& Biobehavioral Reviews, 37(6), 1008-1015.

Chung, Y. B., Williams, W., \& Dispenza, F. (2009). Validating work discrimination and coping strategy models for sexual minorities. The Career Development Quarterly, 58(2), 162-170.

Cohen, B. \& Wysocky, L. (2008). Front of the class: How Tourette Syndrome made me the teacher I never had. New York, N.Y.: Griffin Publishing.

Cohen, S. C., Leckman, J. F., \& Bloch, M. H. (2013). Clinical assessment of Tourette syndrome and tic disorders. Neuroscience \& Biobehavioral Reviews, 37(6), 997-1007.

Colton, A., \& Pistrang, N. (2004). Adolescents' experiences of inpatient treatment for anorexia nervosa. European Eating Disorders Review, 12, 307-316. 
Conelea, C. A., Woods, D. W., Zinner, S. H., Budman, C. L., Murphy, T. K., Scahill, L. D., .. . Walkup, J. T. (2013). The impact of Tourette syndrome in adults: Results from the Tourette syndrome impact survey. Community Mental Health Journal, 49(1), 110-120.

Cooley, C. H. (1922). Human nature and the social order. $2^{\text {nd }}$ edn. New York: Charles Scribner's Sons.

Crabtree, J. W., Haslam, S. A., Postmes, T., \& Haslam, C. (2010). Mental health support groups, stigma, and self-esteem: Positive and negative implications of group identification. Journal of Social Issues, 66(3), 553-569.

Cutler, D., Murphy, T., Gilmour, J., \& Heyman, I. (2009). The quality of life of young people with Tourette syndrome. Child: Care, Health and Development, 35(4), 496-504.

Elliott, R., Fischer, C. T., \& Rennie, D. L. (1999). Evolving guidelines for publication of qualitative research in psychology and related fields. The British Journal of Clinical Psychology, 38(3), 215-229.

Evans, J., Seri, S., \& Cavanna, A. E. (2016). The effects of Gilles de la Tourette syndrome and other chronic tic disorders on quality of life across the lifespan: A systematic review. European Child \& Adolescent Psychiatry, 25(9), 939-948.

Festinger, L. (1954). A theory of social comparison processes. Human Relations, 7(2), 117-140.

Fingerman, K. L. (2009). Consequential strangers and peripheral ties: The importance of unimportant relationships. Journal of Family Theory \& Review, 1, 69-86.

Finlay, W. M. L., \& Lyons, E. (2005). Rejecting the label: A social con- structionist analysis. Mental Retardation, 43, 120-134. https://doi.org/10.1352/0047-6765(2005)432.0.CO;2.

Flowers, P., Marriott, C., \& Hart, G. (2000). The bars, the bogs and the bushes': The impact of locale on sexual cultures. Culture, Health and Sexuality, 2, 69-86.

Forrester-Jones, R., \& Barnes, A. (2008). On being a girlfriend not a patient: The quest for an acceptable identity amongst people diagnosed with a severe mental illness. Journal of Mental Health, 17(2), 153172.

Forrester-Jones, R., \& Grant, G. (1997). Resettlement from large psychiatric hospital to small community residence: One step to freedom. Aldershot: Ashgate Publishing Group.

Freeman, R. D., Fast, D. K., Burd, L., Kerbeshian, J., Robertson, M. M., \& Sandor, P. (2000). An international perspective on Tourette syndrome: Selected findings from 3500 individuals in 22 countries. Developmental Medicine and Child Neurology, 42(7), 436-447.

Goffman, E. (1963). Stigma: Notes on a spoiled identity. Jenkins, JH \& Carpenter.

Guba, E. G., \& Lincoln, Y. S. (1989). Fourth generation evaluation. Newbury Park: Sage.

Grady C. (2005). Payment of clinical research subjects. The Journal of clinical investigation, 115(7), 16811687. https://doi.org/10.1172/JCI25694.

Hanks, C. E., McGuire, J. F., Lewin, A. B., Storch, E. A., \& Murphy, T. K. (2016). Clinical correlates and mediators of self-concept in youth with chronic tic disorders. Child Psychiatry \& Human Development, 47(1), 64-74.

Himle, M. B., Wellen, B. C., \& Hayes, L. P. (2018). Family issues associated with tics. In The clinician's guide to treatment and management of youth with Tourette syndrome and tic disorders (pp. 301-325). Elsevier.

HM Government (2001). Valuing people, a new strategy for learning disability for the 21 st century. UK Department of Health and Social Care.

HM Government. (2009). Valuing people now: A new three-year strategy for learning disabilities. UK Department of Health.

Hollenbeck, P. (1999). How life imitates Tourette syndrome: Reflections of an afflicted neuroscientist. CNS Spectrums, 4(2), 22-23.

Holt, A. (2010). Using the telephone for narrative interviewing: A research note. Qualitative Research, 10(1), $113-121$.

Hubka, G. B., Fulton, W. A., Shady, G. A., Champion, L. M., \& Wand, R. (1988). Tourette syndrome: Impact on Canadian family functioning. Neuroscience \& Biobehavioral Reviews, 12(3-4), 259-261.

Ilic, M., Reinecke, J., Bohner, G., Röttgers, H., Beblo, T., Driessen, M., . . Corrigan, P. W. (2014). Managing a stigmatized identity_Evidence from a longitudinal analysis about people with mental illness. Journal of Applied Social Psychology, 44(7), 464-480.

Jetten, J., Haslam, C., \& Haslam, S. A. (2012). In J. Jetten, C. Haslam, \& S. A. Haslam (Eds.), The case for a social identity analysis of health and well-being. The social cure: Identity, health and well-being. New York, NY, US: Psychology Press.

Khoury, R. (2010). Perceptions of FQOL of families who have a child with Tourette syndrome, Ph. D. Anglia Ruskin University.

Knight, T., Steeves, T., Day, L., Lowerison, M., Jette, N., \& Pringsheim, T. (2012). Prevalence of tic disorders: A systematic review and meta-analysis. Pediatric Neurology, 47(2), 77-90. 
Leckman, J. F., Bloch, M. H., Sukhodolsky, D. G., Scahill, L., \& King, R. A. (2013). Phenomenology of tics and sensory urges: The self under siege. Tourette syndrome (pp. 3-25). New York: Oxford University Press.

Lincoln, Y. S., \& Guba, E. G. (1985). Naturalistic inquiry. In Naturalistic inquiry. Beverly Hills, CA: Sage Publications.

Link, B. G., \& Phelan, J. (2014). Stigma power. Social Science \& Medicine, 103, 24-32.

Link, B. G., Mirotznik, J., \& Cullen, F. T. (1991). The effectiveness of stigma coping orientations: Can negative consequences of mental illness labelling be avoided? Journal of Health and Social Behavior, 32(3), 302-320.

Lucksted, A., Drapalski, A., Calmes, C., Forbes, C., DeForge, B., \& Boyd, J. (2011). Ending self-stigma: Pilot evaluation of a new intervention to reduce internalized stigma among people with mental illness. Psychiatric Rehabilitation Journal, 35, 51-54.

Major, B., \& Schmader, T. (2018). Stigma, Social Identity Threat, and Health. In (Ed.), The Oxford Handbook of Stigma, Discrimination, and Health (pp. 85-103). New York :Oxford University Press.

Malli, M. A., \& Forrester-Jones, R. (2017). 'I'm not being rude, I'd want somebody normal": Adolescents' perception of their peers with Tourette's syndrome: An exploratory study. Journal of Developmental and Physical Disabilities, 29(2), 279-305.

Malli, M. A., Forrester-Jones, R., \& Murphy, G. (2016). Stigma in youth with Tourette's syndrome: A systematic review and synthesis. European Child \& Adolescent Psychiatry, 25(2), 127-139.

Mansley, C. (2003). Making allowances: Autobiographical accounts of people with Tourette syndrome. Liverpool: The Bluecoat Press.

Matsuda, N., Kono, T., Nonaka,M., Fujio,M., \& Kano, T. (2016) Self-initiated coping with Tourette's syndrome: Effect of tic suppression on QOL. Brain and Development 38 (2), 233-241.

McCarthy, M., Hunt, S., \& Milne-Skillman, K. (2017). 'I know it was every week, but I Can't be sure if it was every Day: Domestic violence and women with learning disabilities. Journal of Applied Research in Intellectual Disabilities, 30, 269-282.

Meisenback, R. J. (2010). Stigma management communication: A theory and agenda for applied research on how individuals manage moments of stigmatized identity. Journal of Applied Communication Research, 38(3), 268-292.

Miller, C. T., \& Kaiser, C. R. (2001). A theoretical perspective on coping with stigma. Journal of Social Issues, 57(1), 73-92.

Miller, C. T., \& Major, B. (2000). Coping with stigma and prejudice. In T. F. Heatherton, R. E. Kleck, M. R. Hebl, \& J. G. Hull (Eds.), The social psychology of stigma (pp. 243-272). New York, NY, US: Guilford Press.

Miller, C. T., Rothblum, E. D., Felicio, D., \& Brand, P. (1995). Compensating for stigma: Obese and nonobese women's reactions to being visible. Personality and Social Psychology Bulletin, 21(10), 1093-1106.

Miller, L. L., Scharf, J. M., Mathews, C. A., \& Ben-Shlomo, Y. (2014). Tourette syndrome and chronic tic disorder are associated with lower socio-economic status: Findings from the a von longitudinal study of parents and children cohort. Developmental Medicine and Child Neurology, 56(2), 157-163.

Monteleone, R., \& Forrester- Jones, R. (2017). 'Disability means, um, dysfunctioning people': A qualitative analysis of the meaning an experience of disability among adults with intellectual disabilities. Journal of Applied Research in Intellectual Disabilities, 30, 301-315.

Murray, C. D., \& Sixsmith, J. (1998). E-mail: A qualitative research medium for interviewing? International Journal of Social Research Methodology, 1(2), 103-121.

O'Connor, K., Janelle, C., Coutu, M., Rouleau, A., Lessard, M., Kirouac, C., . . Bélanger, M. (2009). I'm cured but...' perceptions of illness following treatment. Journal of Health Psychology, 14(2), 278-287.

Pillow, W. (2003). Confession, catharsis, or cure? Rethinking the uses of reflexivity as methodological power in qualitative research. International Journal of Qualitative Studies in Education, 16(2), 175-196.

Quinn, D. M., Williams, M. K., Quintana, F., Gaskins, J. L., Overstreet, N. M., Pishori, A., ... \& Chaudoir, S. R. (2014). Examining effects of anticipated stigma, centrality, salience, internalization, and outness on psychological distress for people with concealable stigmatized identities. PloS one, 9(5), e96977.

Reilly, D. E., Huws, J. C., Hastings, R. P., \& Vaughan, F. L. (2008). 'When your child dies you don't belong in that world any more'-experiences of mothers whose child with an intellectual disability has died. Journal of Applied Research in Intellectual Disabilities, 21(6), 546-560.

Rossman, G. B., \& Rallis, S. F. (2003). Learning in the field: An introduction to qualitative research. Sage.

Sacks, O. (1995). An anthropologist on Mars: seven paradoxical tales. New York: Knopf.

Schneider, J.W. (2009). Having epilepsy: the experience and control of illness. Philadelphia, Pennsylvania, USA: Temple University Press. 
Shinebourne, P. \&. Smith, J.A. (2009) Alcohol and the self: An interpretative phenomenological analysis of the experience of addiction and its impact on the sense of self and identity, Addiction Research \& Theory, $17: 2,152-167$.

Smith, J. A. (1996). Evolving issues for qualitative psychology. In J.T.E Richardson (Ed), Handbook of Qualitative Research Methods for Psychology and the Social Sciences, (pp. 189-201). Leicester, UK: British Psychological Society.

Smith, J. A., \& Osborn, M. (2007). Pain as an assault on the self: An interpretative phenomenological analysis of the psychological impact of chronic benign low back pain. Psychology \& Health, 22(5), 517-534.

Smith, J. A., Flowers, P., \& Larkin, M. (2009). Interpretative phenomenological analysis. Theory, method and research. London: Sage.

Smith, H., Fox, J. R., \& Trayner, P. (2015). The lived experiences of individuals with Tourette syndrome or tic disorders: A meta-synthesis of qualitative studies. British Journal of Psychology, 106(4), 609-634.

Steele, C. M., Spencer, S. J., \& Aronson, J. (2002). In M. P. Zanna (Ed.), Contending with group image: The psychology of stereotype and social identity threat. Advances in experimental social psychology (pp. 379-440), San Diego, CA, US: Academic Press.

Sue, D. W. (2003). Overcoming our racism: The journey to liberation. Hoboken, NJ: John Wiley \& Sons.

Sue, D. W. (2010). Microaggressions and marginality: Manifestation, dynamics, and impact. Hoboken, NJ: John Wiley \& Sons.

Swain, J. E., Scahill, L., Lombroso, P. J., King, R. A., \& Leckman, J. F. (2007). Tourette syndrome and tic disorders: A decade of progress. Journal of the American Academy of Child \& Adolescent Psychiatry, 46(8), 947-968.

Tajfel, H. (1972). Experiments in a Vacuum). In J. Israel \& H. Tajfel (Eds.), The context of social psychology: A critical assessment (pp. 69-119). London: Academic Press.

Tajfel, H. (1974). Social identity and intergroup behaviour. Social Science Information/sur les sciences sociales, 13(2), 65-93.

Tajfel, H., \& Turner, J. C. (1979). An Integrative Theory of Intergroup Conflict. In W. G. Austin, \& S. Worchel (Eds.), The Social Psychology of Intergroup Relations (pp. 33-47). Monterey, CA: Brooks/Cole.

Todd, S., \& Shearn, J. (1997). Family dilemmas and secrets: Parents' disclosure of information to their adult offspring with learning disabilities. Disability \& Society, 12(3), 341-366.

Turner, J. C. (1982). Towards a cognitive redefinition of the social group. In H. Tajfel (Ed.), Social identity and intergroup relations (pp. 15-40). Cambridge : Cambridge University Press.

Wadman, R., Tischler, V., \& Jackson, G. (2013). Everybody just thinks I'm weird': A qualitative exploration of the psychosocial experiences of adolescents with Tourette syndrome. Child: Care, Health and Development, 39(6), 880-886.

Wills, T. A. (1981). Downward comparison principles in social psychology. Psychological Bulletin, 90(2), 245-271.

World Health Organization, \& WHO. (1992). The ICD-10 classification of mental and behavioural disorders: Clinical descriptions and diagnostic guidelines World Health Organization.

Publisher's Note Springer Nature remains neutral with regard to jurisdictional claims in published maps and institutional affiliations.

\title{
Affiliations
}

\author{
Melina Aikaterini Malli ${ }^{1} \cdot$ Rachel Forrester-Jones $^{2} \cdot$ Paraskevi Triantafyllopoulou $^{1}$ \\ Tizard Centre, University of Kent, Canterbury CT2 7NF, UK \\ 2 Department of Social \& Policy Sciences, University of Bath, Cleverton Down, Bath BA2 7AY, UK
}

